\section{La reformulación de la política exterior argentina en el gobierno de Macri: el difícil equilibrio entre los cambios globales y las crisis domésticas (2015-2018)*}

Anuario Latinoamericano Ciencias Políticas y Relaciones Internacionales vol. 7, 2019

pp. 193-212

DOI: $10.17951 /$ al.2019.7.193-212

\section{The Reformulation of Argentina's Foreign Policy under the Government of Macri: the Difficult Balance between Global Changes and Domestic Crises (2015-2018)}

\author{
Priscila Palacio ${ }^{\star *}$ \\ FACULTAD DE CIENCIAS ECONÓMICAS \\ UNIVERSIDAD DE BUENOS AIRES, ARGENTINA \\ $\triangle$ prisilun@gmail.com \\ https://orcid.org/0000-0002-9773-4160
}

\section{RESUMEN}

El trabajo analiza la política exterior de Macri, argumentando que factores domésticos conllevaron a cambiar el posicionamiento internacional que había adoptado Argentina con C. Kirchner. Pero los cambios globales parecen imponer un pragmatismo que contradice el idealismo macrista a la vez que la crisis de la economía argentina dificultó consolidar el nuevo paradigma. El marco de análisis es el realismo clásico, incorporando el rol de los factores domésticos en la política mundial (Gilpin 1981). El estudio principia con un planteo teórico; luego se exponen acontecimientos mundiales que denotan un auge del proteccionismo en el siglo XXI. Finalmente, se analiza la politica exterior de Macri, destacando las restricciones que impuso la economía doméstica.

PALABRAS CLAVE: política exterior argentina, cambios globales, realismo clásico, política económica argentina.

* El presente artículo es parte de las investigaciones desarrolladas en el marco del Proyecto de Investigación UBACYT "Cambios domésticos (económicos y políticos) de Argentina vis a vis los cambios de paradigmas mundiales: actores de poder, diplomacia, ciclos y restricciones estructurales (1989-2016). Estudio integral desde la Economía Política Internacional y la Historia", dirigido por la Prof. Dra. Priscila Palacio.

** Profesora e Investigadora de la Facultad de Ciencias Económicas, Universidad de Buenos Aires. Doctora en Relaciones Internacionales. Magíster en Relaciones Económicas Internacionales. Contador Público. Integrante del Centro de Estudios Latinoamericanos para el Desarrollo y la Integración (FCE-UBA). 


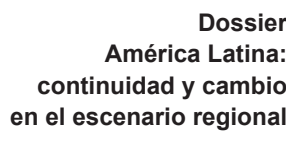

América Latina: en el escenario regional

\begin{abstract}
This paper analyzes Macri's foreign policy, arguing that domestic factors led to the change of the international positioning that Argentina had got under C. Kirchner's government. But global changes seem to impose pragmatism that contradicts the Macri's idealism, while the crisis in the Argentine economy made it difficult to consolidate the new paradigm. The framework of analyzes is classical realism-incorporating the role of domestic factors in world politics (Gilpin 1981). The study begins with a theoretical approach; then world events are presented to denote a boom in protectionism in the $21^{\text {st }}$ century. Finally, Macri's foreign policy is analyzed, highlighting the restrictions imposed by the domestic economy.
\end{abstract}

KEYWORDS: Argentina's foreign policy, global changes, classical realism, Argentine economic policy.

\section{Introducción}

El trabajo presenta un abordaje de la política exterior argentina durante el gobierno de Mauricio Macri, proponiendo un análisis desde la perspectiva del realismo clásico en relaciones internacionales. Se pretende mostrar que, más allá de las estructuras y dinámicas a nivel internacional, inciden factores domésticos ${ }^{1}$ en la definición de política exterior de los Estados -aun cuando se trate de países que no revisten la calidad de potencias, como es el caso de Argentina.

En la primera sección se exponen los ejes del marco teórico, basado en el enfoque de Robert Gilpin (1981) -que destaca el rol de los factores domésticos en la política mundial. Se parte de la premisa que Gilpin provee un marco de análisis que retoma el realismo clásico, permitiendo comprender la complejidad de variables que intervienen en las dinámicas internacionales -tanto sean internas, como externas (Palacio 2019). Su enfoque, claramente, se distancia del realismo estructuralista que ha predominado en el pensamiento realista de las últimas décadas.

En la segunda sección, se analizan algunos acontecimientos que denotan un replanteo del paradigma económico imperante a nivel mundial, en el siglo XXI. Se indaga, brevemente, en las causas que han generado el resurgimiento del proteccionismo o nacionalismo económico ${ }^{2}$. Estas dos tenden-

1 Como la ideología del grupo gobernante y el devenir de la economía nacional.

2 El nacionalismo económico es uno de los tres modelos que puede asumir la economía política de un país (Gilpin 1990). En ese modelo, el Estado desempeña un rol fundamental en la administración económica y la seguridad nacional. Si bien hay distintos tipos y grados de proteccionismo económico - siendo el más extremo la autarquía, generalmente, el modelo consiste en el establecimiento de barreras arancelarias y/o no arancelarias, a fin de reducir las importaciones y fomentar el desarrollo de la industria nacional. De ese modo, se busca disminuir el déficit, equilibrar u obtener superávit en la balanza comercial. 
cias han surgido para frenar el impacto de la globalización ${ }^{3}$ y neoliberalismo institucional ${ }^{4}$.

Finalmente, en la última sección, se estudian los aspectos más relevantes de la política exterior del gobierno de Macri, entre 2015 y $2018^{5}$-período en el cual se definieron los ejes fundamentales de su gestión política. El propósito es evidenciar que los factores domésticos (los actores de poder y la ideología del grupo gobernante) propiciaron el cambio de paradigma en el posicionamiento internacional que había adquirido Argentina bajo la gestión de Cristina F. de Kirchner. Sin embargo, esa reformulación de la política exterior argentina en el gobierno macrista planteó un equilibrio difícil de lograr. Por un lado, los cambios globales (nuevo auge del proteccionismo) parecen ir a contramano de la retórica idealista ${ }^{6}$, que pretendió materializar la gestión de Macri; por el otro, las restricciones que impuso la crisis de la economía doméstica han dificultado la consolidación del nuevo paradigma económico en el ámbito nacional.

\section{Enfoque teórico: el realismo clásico}

Desde una perspectiva que retoma los principios del realismo clásico, Gilpin (1981) plantea que el carácter de una sociedad es crítico para determinar su respuesta ante modificaciones en la distribución de poder internacional. $\mathrm{Su}$ enfoque se distancia de los postulados del realismo estructuralista -o neorrealismo ${ }^{7}$, ya que este último hace abstracción de los factores domésticos en el nivel de análisis sistémico. De hecho, Mastanduno (2012) recuerda que, mientras Gilpin exhibía un gran respeto académico por Kenneth Waltz, encontraba al neorrealismo demasiado restrictivo:

3 Aunque existen innumerables definiciones de la globalización, en el presente trabajo se considera a la misma como el proceso de profundización de la interrelación de la economía de un país con el resto del mundo. Este proceso requiere de la liberalización y desregulación de las economías nacionales, con el propósito de facilitar la integración de los mercados a nivel mundial, promover la libre circulación de factores e incrementar las transacciones comerciales, financieras y de servicios en el sistema internacional.

4 Desde el punto de vista económico, el neoliberalismo institucional se asocia con los principios de la interdependencia compleja y el avance de la globalización -véase Rourke (2007) y Cohen (2008).

5 Su actual gestión de gobierno se extiende hasta diciembre de 2019.

6 En relaciones económicas internacionales, el idealismo -término que, como señala Rourke (2007), cayó en desuso- se asocia con los principios del liberalismo económico. Como sostiene Gilpin (1990: 39-43), a lo largo de los años, el liberalismo ha asumido diversas formas, pero todas ellas consideran al mercado y al mecanismo de precios como el medio más eficaz para organizar las relaciones económicas internas e internacionales. Asimismo, los liberales creen que el comercio y el intercambio generan relaciones pacíficas entre los países porque los beneficios del comercio y la interdependencia favorecen la cooperación.

7 Entre sus principales referentes figuran Waltz (1979) y Mearsheimer (2001)
La reformulación de la política exterior argentina en el gobierno de Macri: el difícil equilibrio entre los cambios globales y las crisis domésticas (2015-2018)

Priscila Palacio 


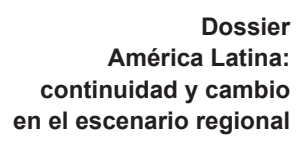

América Latina: en el escenario regional
... [Gilpin] forzaba a los estudiantes en los seminarios a reconocer que las ideas importan tanto como el poder material [...] y que cualquier análisis serio de relaciones internacionales necesitaba un marco para integrar las variables políticas domésticas. (Mastanduno 2012: 9, trad. propia)

Aunque ese criterio de análisis holístico ya era reconocido por el realismo clásico, en las últimas décadas se vio sometido a crítica por la corriente estructuralista -que adquirió prevalencia en la producción de teoría realista desde los años ochenta, centralizando el estudio en el nivel del sistema internacional.

Los realistas estructuralistas ignoran las diferencias culturales entre los Estados tanto como las diferencias en el tipo de régimen, principalmente debido a que el sistema internacional crea los mismos incentivos básicos para todos los grandes poderes [...] No importa quién está a cargo de conducir la política exterior del Estado [...] Los realistas estructuralistas tratan a los Estados como si fueran cajas negras. (Mearsheimer 2006: 72, trad. propia)

Contrario al estructuralismo, el enfoque de Gilpin (1981) sostiene que Estado y sociedad están compuestos por individuos y grupos diferenciables que se influencian mutuamente. Los grupos poderosos establecen restricciones y pueden determinar las acciones de las autoridades estatales:

[...] aún el dictador más despiadado debe satisfacer los intereses de esos individuos y grupos que también ejercen el poder en una sociedad. Los grupos poderosos [...] constituyen la sociedad que es protegida por el Estado; su particular concepto de justicia reina. (Gilpin 1981: 16, trad. propia)

Siguiendo la definición de Pareto, esos grupos dominantes en la economía y el gobierno son las élites, que asignan recursos y ejercen el control del proceso de influencia (Fernández Pardo 2015: 46). Asimismo, el rol que Gilpin (1981) asigna a los factores domésticos en la dinámica de las relaciones interestatales, hace que su enfoque satisfaga las aclamaciones por la flexibilización del realismo estructuralista que varios académicos han efectuado desde hace algunos años. Tal es el caso de Snyder (1991) quien, con su teoría de las coaliciones y el tráfico de influencias, concluye que el realismo debe ser "recapturado" de aquellos que miran solamente a la política entre las sociedades, ignorando lo que ocurre dentro de ellas. Inclusive, dicho autor señala que algunos exponentes del realismo, refiriéndose a Waltz (1979), se han equivocado en tomar a los Estados como "átomos irreducibles". En un sentido similar se pronuncia Kirshner (2010: 42-4), al señalar las limitaciones del neorrealismo waltziano para analizar el siglo XXI -considerando a los Estados como unidades diferenciadas solamente por sus capacidades relativas. Pero, como se expresó, 
Gilpin ya se apartaba del neorrealismo de Waltz, al sostener que hay múltiples determinantes domésticos de la política exterior de los Estados.

Hay simplemente demasiadas variables cualitativas: personalidades, carácter nacional, estructura social, intereses económicos, organización política, etc. Es más, a medida que estos factores cambian, también lo hacen los intereses y el poder de los Estados en sí mismos [...] Si estos cambios domésticos fomentarán a un Estado a expandirse territorialmente, caer en aislamiento, o intentar alterar la división internacional del trabajo sólo puede ser determinado por el registro histórico. (Gilpin 1981: 96-7, trad. propia)

Además, señalaba que hay patrones que determinan la conexión entre el factor doméstico y el cambio internacional. Entre ellos, su enfoque destaca la relación entre ganancia pública y privada, sosteniendo que el principal mecanismo de reconciliación de los beneficios o costos sociales y privados es la definición de derechos de propiedad de la sociedad (Gilpin 1981: 104). De ese modo, Gilpin reconocía el rol de los actores de poder y el tráfico de influencias en la definición de la política exterior de los Estados.

Finalmente, su enfoque se acerca a los parámetros que sustenta el realismo clásico (Palacio 2019), permitiendo comprender con mayor grado de "realismo" las relaciones internacionales en un mundo interconectado y absolutamente penetrado por el avance tecnológico.

\section{Indicios de un cambio de paradigma económico mundial en el siglo XXI}

Es indudable que los cambios globales se han profundizado desde la asunción de Donald Trump a la presidencia de los Estados Unidos de América, en enero de 2017. Sin embargo, la gestación de esta nueva ola que cuestiona la globalización y el neoliberalismo institucional proviene de larga data (Arceo 2016). Posiblemente, el origen de estos cambios se remonta al segundo lustro de la década de 1990, es decir, están asociados a las consecuencias sociales de la globalización económico-financiera.

Al igual que sucedió con otros fenómenos de la historia económica contemporánea, el avance de la globalización -entre la década de 1970 y comienzos del XXI- no fue ni unívoco ni homogéneo. Coetáneo a su auge, se registró durante los años noventa la proliferación de acuerdos de integración regional, los cuales partieron el comercio mundial en bloques discriminatorios. El fin de la Guerra Fría dinamizó la concertación y/o revitalización de dichos acuer$\operatorname{dos}^{8}$. El propósito fue lograr una mayor desregulación económica -siguiendo el paradigma recopilado en el Consenso de Washington. El neoliberalismo se

\footnotetext{
8 Algunos de los acuerdos regionales tenían sus antecedentes en la segunda posguerra.
}

La reformulación de la política exterior argentina en el gobierno de Macri: el difícil equilibrio entre los cambios globales y las crisis domésticas (2015-2018)

Priscila Palacio 
Dossier América Latina: continuidad y cambio en el escenario regional fue plasmando, de un modo u otro, en el acervo normativo de muchos de los acuerdos que se firmaron por esos años, llevando a acuñar el término regionalismo abierto. Para algunos autores, globalización y regionalización eran dos facetas de un mismo fenómeno; otros, en cambio, sostenían que la regionalización era el paso previo a la globalización de las economías nacionales.

En el ámbito multilateral, las rondas de negociaciones en el GATT (General Agreement on Tariffs and Trade) permitieron significativos progresos en la liberalización del comercio. Pero el logro más trascendente se concretó en la Ronda Uruguay, con la creación de la Organización Mundial del Comercio (OMC), a finales de $1994^{9}$. Sin embargo, al tiempo que la globalización alcanzaba su cenit, empezaron a explicitarse los efectos adversos del proceso globalizador. La cara más dramática fueron las crisis financieras internacionales que -a través del denominado "efecto contagio"- golpearon a las economías emergentes, en la segunda mitad de los años noventa ${ }^{10}$. Tales crisis se propagaron al "estilo dominó", e incluyeron la "crisis del tequila" (México 1994/95), la crisis asiática (Tailandia 1997), la crisis rusa (Rusia, 1998), la crisis brasileña (Brasil, 1998/99), la crisis turca (Turquía, 2001) y la crisis argentina (Argentina, 2001). Los países víctimas de los movimientos de capitales especulativos habían desregulado sus economías -siguiendo las pautas recomendadas por el FMI (Palacio 2008: 166-7).

Las crisis de los países emergentes no fueron sólo de carácter financiero, sino económico. Ellas dejaron al descubierto las profundas fragilidades económico-sociales, que existían en los países afectados. En el ámbito doméstico, se había producido una expansión de los sectores marginales y excluidos del mercado laboral formal. Ese fue el germen de los denominados movimientos antiglobalización que salieron a la luz a comienzos del nuevo siglo, reivindicando el accionar del Estado-nación frente a la globalización.

Desde el punto de vista económico, es indiscutible que, en las últimas décadas del siglo XX, el mundo experimentó una revolución en la economía y las finanzas mundiales que se aceleró durante los años noventa. El extraordinario crecimiento económico posibilitó dejar atrás varios lustros de estancamiento y recesión -que afectó a las economías centrales y en desarrollo, tras la crisis de la estanflación (iniciada en 1973).

La expansión consensuada del liberalismo fue uno de los factores que impulsó el crecimiento de la economía mundial. Sin embargo, desde el punto de vista relativo, las más favorecidas fueron algunas grandes economías, como es el caso de EE. UU, y ciertos mercados emergentes, como ocurrió con China e India. Éstos últimos tuvieron acceso a ingentes cantidades de capitales atraídos por la desregulación de sus mercados y las altas tasas de crecimiento de sus

\footnotetext{
9 Con vigencia a partir del año 1995.

10 El fenómeno de contagio provoca que los países que presentan características económicas similares a un país que está enfrentando una crisis financiera, sean considerados como países "de alto riesgo" por parte de los inversores internacionales, sufriendo una masiva retirada de capitales.
} 
respectivas economías. De ese conjunto de los países emergentes, el país cuyo PBI creció de manera más acelerada y sostenida fue indudablemente China ${ }^{11}$.

Empero, como se mencionó anteriormente, una paradoja de la globalización fueron los efectos devastadores sobre quienes quedaron al margen del progreso anhelado. La abrupta imposición del liberalismo -tanto en economías maduras como en las en desarrollo- produjo la liquidación de vastos sectores económicos, incapaces de competir con importaciones liberadas. Algunas manifestaciones contra la globalización empezaron a surgir en el ámbito local ${ }^{12}$, incluso antes de su debut en el corazón del sistema económico mundial.

La primera gran manifestación internacional antiglobalización tuvo lugar en Seattle (1999), en ocasión de celebrarse la "Ronda del Milenio" de la OMC. La misma convocó a miles de manifestantes de diversos países, pero estuvo liderada por organizaciones sindicales estadounidenses -voceras de los perdedores de la competencia desleal que propiciaba la liberalización económica (Frieden 2006: 457-9).

La repercusión de la protesta fue evidente. Unos días después, los periódicos del mundo anunciaban el rotundo fracaso de las negociaciones en la "Ronda del Milenio". Seattle fue la primera de una serie de manifestaciones internacionales que adquirieron notoriedad en ocasión de celebrarse los grandes encuentros de foros y organismos internacionales. A ella le siguieron las protestas de Gotemburgo (2001), Génova (2001) y Porto Alegre (2002), entre otras. Como, oportunamente, reconoció el propio FMI:

El término "globalización" ha adquirido una fuerte carga emotiva. Algunos consideran que la globalización es un proceso beneficioso -una clave para el desarrollo económico futuro en el mundo-, a la vez que inevitable e irreversible. Otros la ven con hostilidad, incluso temor, debido a que consideran que suscita una mayor desigualdad dentro de cada país y entre los distintos países, amenaza el empleo y las condiciones de vida y obstaculiza el progreso social. (FMI 2000)

Aunque el FMI reconocía las limitaciones de la globalización para disminuir la desigualdad social, destacaba que el siglo XX registró un crecimiento económico sin precedentes. Pero admitía que el crecimiento había sido inequitativo. Para la cuarta parte de la población mundial más rica, el PIB per cápita casi se sextuplicó en el siglo XX; para la cuarta parte más pobre no llegó a triplicarse (FMI 2000). Pese a ello, señalaba que:

${ }^{11}$ La sigla BRIC fue introducida por Jim O 'Neill, jefe de Investigación Económica Global de Goldman Sachs, para referirse a las economías emergentes de Brasil, Rusia, India y China, cuyo crecimiento del PBI en 2001-2002 excedería el de los países del G7, y cuyo PBI hacia fines del año 2000 equivaldría al $23 \%$ del producto mundial (O'Neill 2001).

12 En 1994, el mundo atestiguó el levantamiento indígena armado autodenominado Ejército Zapatista de Liberación Nacional (Chiapas, México), que se pronunció en contra del neoliberalismo (Olesen 2004: 89).
La reformulación de la política exterior argentina en el gobierno de Macri: el difícil equilibrio entre los cambios globales y las crisis domésticas (2015-2018)

Priscila Palacio 


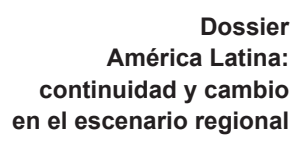

América Latina: en el escenario regional
[... el el ingreso no lo explica todo; una medición más amplia del bienestar que tiene en cuenta las condiciones sociales muestra que los países más pobres han logrado considerables avances [...] si se comparan los países utilizando los indicadores del desarrollo humano (IDH) elaborados por las Naciones Unidas, que tienen en cuenta la educación y la esperanza de vida, el panorama es muy diferente del que muestran los datos referidos solamente al ingreso [...]. (FMI 2000)

Aun así, el FMI (2000) cuestionaba las críticas de ciertos analistas respecto a que las crisis financieras eran el resultado directo e inevitable de la globalización ${ }^{13}$. Para el FMI, si las economías emergentes no hubiesen tenido acceso a los mercados mundiales de capitales, las crisis no se habrían producido en el mismo grado, pero sin esos flujos de capital tampoco se hubiesen registrado las excepcionales tasas de crecimiento que se evidenciaron en la última década del siglo XX.

En los primeros años de la década dos mil, los cuestionamientos a la globalización prosiguieron. En algunas economías en desarrollo y emergentes, fueron acompañados de un resurgimiento de demandas nacionalistas y proteccionistas, sobre todo en aquellas afectadas por las crisis de los años noventa. Tal situación se constituyó en un obstáculo para que EE. UU. pudiera concretar su proyecto de crear una zona de libre comercio en América: el Área de Libre Comercio de las Américas (ALCA).

Pero más allá de la resistencia regional, dos acontecimientos fueron cruciales para que Estados Unidos decidiera empezar a desandar la senda de liberalización comercial. Por un lado, los ataques terroristas del 11 de septiembre de 2001 (11-S) que conmovieron al mundo en los albores del presente siglo. Múltiples fueron los cambios en materia de política, normativa y sociedad, que empezó a implementar EE. UU. a raíz del ataque, sobre todo en temas migratorios y de seguridad nacional. Por el otro, la crisis internacional del 2008, que se originó en la economía de dicho país. Comenzó como un pánico financiero, a mediados de septiembre de 2008, y provocó una estrepitosa caída de la bolsa de valores estadounidense tras conocerse la declaración de bancarrota del banco Lehman Brothers ${ }^{14}$. La crisis bursátil marcó el inicio de un período de recesión a nivel mundial (Palacio 2012).

La crisis desatada en los EE. UU. se propagó por efectos de la globalización financiera. Nuevamente, operó el "efecto contagio" que, durante las crisis de los años noventa, había golpeado a las economías emergentes. La novedad de este nuevo contagio fue que quienes compartían características de vulnerabilidad eran las economías europeas. A ellas no sólo les afectó el bajo crecimiento, sino la revaluación del euro ${ }^{15}$.

${ }^{13}$ Un interesante análisis en defensa de los logros de la globalización puede hallarse en Bhagwati (2004).

${ }^{14}$ Se trataba del cuarto -en orden de importancia- banco de inversiones de EE.UU., con una estructura de más de 29.000 empleados alrededor del mundo.

15 Algunas economías europeas evidenciaban un incremento en la morosidad de su mercado hipotecario -tal como sucedió en España, Francia y el Reino Unido. 
En el caso de economías latinoamericanas y otros mercados emergentes, si bien se vieron afectados por la volatilidad en los mercados de valores, las repercusiones fueron menores. Pese a ello, se demostró errónea la tesis del desacople difundida por economistas del FMI -que formulaba la posibilidad de que las economías en desarrollo estuvieran desacopladas de la evolución de las economías desarrolladas (Ocampo 2009: 10).

La otra novedad de la crisis desatada en el 2008 es que vino a mostrar la fragilidad de un modelo económico, en el que se otorgó a las entidades financieras el poder mágico de creadores de riqueza - a través de los nuevos instrumentos financieros-, siendo estas meras instituciones de intermediación [...]. (Palacio 2012: 123)

Como consecuencia de la sucesión de crisis asociadas a la globalización, muchas economías, incluida la estadounidense, retomaron políticas intervencionistas. Además, se observó el resurgimiento de demandas por políticas benefactoras. El proteccionismo emergió una vez más como respuesta cíclica a los embates del liberalismo económico.

Tal como se mencionó anteriormente, Estados Unidos no fue el único afectado. Desde 2011, se difundió por distintos países de Europa el movimiento de los “indignados" -que nació el 15 de mayo (15M) en España, y se trasladó a Grecia, Francia, Italia, Alemania, entre otros países. Miles de manifestantes, en su mayoría jóvenes convocados por redes sociales, se pronunciaban en contra de las medidas económicas de ajuste, en contra del desempleo y los vicios de la democracia (Corradini 2011). La ola de protestas se inspiró en las sublevaciones que habían dado lugar a la denominada (eufemísticamente) Primavera Árabe, y se extendió de manera intermitente, con consecuencias diversas, por los diferentes países de la vieja Europa:

Desde el comienzo de la crisis en 2008, nuestros gobernantes decidieron poner de rodillas a los pueblos, en vez de castigar a los bancos. Las democracias europeas han sido secuestradas por los mercados financieros. Estamos asfixiados por los planes de austeridad que se multiplican en Europa", reza la proclama lanzada el 22 de mayo por una asamblea general en la Bastilla. (Corradini 2011)

Asimismo, en marzo de 2016, otro importante acontecimiento vino a replantear el orden institucionalista heredado de la segunda posguerra. Se trató del referéndum que llevó adelante el Reino Unido e Irlanda del Norte, para votar la salida de ese país de la Unión Europea (BREXIT). Aunque mayoritariamente, los votantes británicos se inclinaron por el abandono de la Unión Europea, fundamentándose en los problemas que acarreaba la facilidad de migración en el ámbito regional (inseguridad y desempleo), entre otros aspectos, la realidad es que, hasta el momento, el Parlamento inglés no pudo consensuar un acuerdo satisfactorio para la salida de la Unión Europea. De hecho, varios
La reformulación de la política exterior argentina en el gobierno de Macri: el difícil equilibrio entre los cambios globales y las crisis domésticas (2015-2018)

Priscila Palacio 


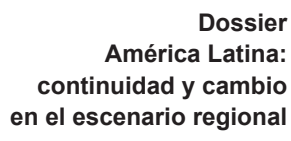

América Latina: el escenario region analistas sostuvieron que el Reino Unido no evaluó correctamente los costos que le acarrearía el abandono del bloque regional.

Finalmente, con el ascenso de Donald Trump a la presidencia de los Estados Unidos se dio un nuevo impulso al movimiento proteccionista a nivel internacional. Trump asumió con la promesa de proteger las industrias estadounidenses, largamente afectadas por su falta de competitividad (respecto a sus pares asiáticas). Incluso, gran parte del apoyo electoral que obtuvo el candidato Trump provino de sectores sociales que habían quedado excluidos del mercado laboral formal por la retracción que sufrió la producción industrial en ese país. Por ello, en gran medida, el ascenso de Trump se explica por la crisis socioeconómica que produjo la globalización en las propias economías desarrolladas.

De modo que, en una coyuntura de avance del proteccionismo y repliegue del liberalismo económico -con argumentos que llevaron a cuestionar la globalización en el propio centro de la economía mundial- parece irrealizable la idea de desaparición del Estado, lo que pretendieron instalar algunos defensores de la globalización. Es más, se observa una evidente tensión entre la ola de creciente realismo (nacionalismo/proteccionismo) versus los intentos de sostener el orden institucionalista liberal del sistema Pos-Bretton Woods que parece cada vez más debilitado. Aunque resulte sorprendente, ese orden liberal está siendo defendido por algunos poderes emergentes, especialmente China, que necesita de la liberalización a escala global para sostener su crecimiento doméstico.

\section{Reformulación de la política exterior argentina en los primeros años del gobierno de Macri (2015-2018)}

Es paradójico afirmar que el gobierno que antecedió a la gestión de Mauricio Macri, el de Cristina Fernández de Kirchner (2007-2015), se anticipó en algunos aspectos a los cambios de paradigma mundial que se mencionaron en la sección precedente. La política exterior de Cristina Kirchner tuvo una impronta nacionalista que respondía a la retórica ideológica que promocionó su espacio político: estimular la protección de la industria sustitutiva de importaciones, en el ámbito doméstico ${ }^{16}$.

Asimismo, desde el punto de vista regional, el gobierno de Cristina Kirchner criticó el liberalismo y profundizó la alianza política con países de la región latinoamericana, sobre todo, en los períodos en que hubo gobiernos afines en el ámbito político/ideológico; tal como ocurrió con Brasil bajo la gestión de Lula da Silva y, especialmente, con Rousseff; Ecuador con Correa; Bolivia con Evo Morales; Venezuela con Chávez y, luego Maduro; y, en menor medida, con Chile -en los períodos de gobierno de Bachelet. Como señalan

\footnotetext{
16 Aunque careció de un programa de desarrollo integral competitivo para el largo plazo.
} 
Tokatlian y Russell (2017), los Kirchner procuraron redefinir con sello propio la identidad internacional del Argentina, volviendo a instaurar modelos que tradicionalmente tuvo el país, como su pertenencia al Sur y cuestionamiento a ciertas normas del orden impuesto por Occidente.

De ese modo, la política exterior de Cristina Kirchner fue más allá del interés en la región, procurando, entre otras medidas, restablecer cuestionadas relaciones con Irán ${ }^{17}$, y acercándose a Rusia y, fundamentalmente, a China ${ }^{18}$, al tiempo que mantuvo su distanciamiento político con los Estados Unidos (incluso, bajo la gestión de Obama).

Esos alineamientos regionales e internacionales fueron severamente criticados en la campaña presidencial de Mauricio Macri, quien propuso "normalizar" las relaciones internacionales de Argentina, reintegrando el país al concierto de naciones de la arena mundial e incrementando la firma de tratados de libre comercio ${ }^{19}$. Ello significaba alejarse de los gobiernos populistas de la región, así como de la cuestionada vinculación con Irán.

De hecho, esos fueron los ejes de las primeras medidas que adoptó Macri en política exterior, al asumir su presidencia en diciembre de 2015. La prioridad de Cambiemos ${ }^{20}$ fue reinsertar a la Argentina en el contexto económico internacional. Para ello, se liberalizaron los controles de cambios -que el gobierno de Cristina Kirchner había endurecido desde el año 2011, con la esperanza de que el país recibiría un ingente flujo de inversiones extranjeras y capitales financieros ("lluvia de inversiones"). Además, con igual propósito, se quitaron y redujeron retenciones al campo ${ }^{21} \mathrm{y}$, meses después, en abril de 2016, Argentina terminó acordando con los holdouts, para levantar las cautelares internacionales que impedían el normal pago de la deuda externa.

Con el pago a los buitres, el Gobierno siguió cosechando elogios del establishment financiero internacional, como el del titular del Tesoro de los Estados Unidos, Jacob Lew, que destacó la "acción decisiva" del Gobierno, que "está dando vuelta una página sobre un período difícil de su historia", en relación con el default. "El regreso de Argentina a los mercados de capitales internacionales y su reintegración con la economía global representa un hito importante no sólo para Argentina sino también para todo el sistema global

17 Fue a través de la propuesta y aprobación por el parlamente argentino del Memorándum con Irán, que tuvo como propósito indagar a los sospechosos del atentado a la AMIA (1994).

${ }^{18}$ Con China se firmaron numerosos acuerdos comerciales y financieros.

19 Entre otros, la incorporación a la Alianza del Pacífico, finalización del acuerdo de libre comercio entre Unión Europea y MERCOSUR, negociación de un tratado de libre comercio con Estados Unidos e incorporación al Tratado de Asociación Transpacífico (Arceo 2016).

${ }^{20}$ Cambiemos es la alianza política que llevó a Macri a la presidencia. Fundamentalmente, está integrada por Propuesta Republicana (PRO), la Unión Cívica Radical y la Coalición CívicaARI, además de otros partidos de menor alcance.

${ }^{21}$ Una promesa de campaña del gobierno fue la rebaja y quita de retenciones al campo, lo cual efectivizó a comienzos de su gestión, en diciembre de 2015 (La Nación 2015).
La reformulación de la política exterior argentina en el gobierno de Macri: el difícil equilibrio entre los cambios globales y las crisis domésticas (2015-2018)

Priscila Palacio 


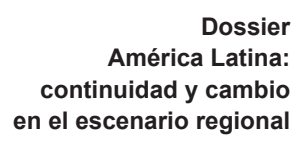

América Latina: en el escenario regional financiero", aseguró el titular del Tesoro, que destacó además la velocidad [... con la] que se llevó adelante el acuerdo. (Valli 2016)

Macri también se preocupó por restablecer las relaciones con el gobierno de los Estados Unidos. Incluso, durante la campaña presidencial de aquel país, en 2016, el gobierno argentino apostó explícitamente al triunfo de la candidatura de Hillary Clinton -lo que algunos analistas consideraron un "grave error de diagnóstico" por parte de la Cancillería argentina, por entonces a cargo de Susana Malcorra. En junio de 2017, poco después del triunfo de Donald Trump en Estados Unidos, la Canciller Malcorra -aludiendo cuestiones personales, fue reemplazada por el Canciller Jorge Faurie.

El jefe de Estado Argentino participó de la asamblea general de la Organización de las Naciones Unidas (ONU), y en sus actividades previas fue parte de la reunión de la Clinton Global Initiative, donde respaldó la carrera presidencial de Hillary, con un contundente mensaje a su marido, Bill.

Al comenzar su exposición, el primer ministro italiano Matteo Renzi bromeó con Clinton, al señalarle: "Mi mujer lo espera en Italia como primer caballero durante el G7”, la cumbre mundial que se realizará el año próximo en la isla italiana de Sicilia.

Macri no se quiso quedar atrás. "Y mi mujer Juliana Awada lo espera como primer caballero en el G20 en Buenos Aires", previsto para el 2018, afirmó el mandatario. (iProfesional 2016)

Pese a ese desliz diplomático, el gobierno argentino pudo reformular rápida y exitosamente las relaciones con la triunfante administración de Donald Trump, basándose en que ambos mandatarios provenían del sector empresarial y en el pasado habían tenido contactos en común, lo que facilitó el mutuo acercamiento. El vínculo con Estados Unidos se transformó en el eje central de la estrategia externa del gobierno de Cambiemos y en la llave de la "vuelta al mundo".

Por otra parte, también desde el inicio de su gestión, Macri se distanció de los gobiernos regionales que sostenían banderas de izquierda o populistas, como Venezuela ${ }^{22}$, Bolivia y Ecuador, acercándose a figuras como Temer (Brasil), Piñera (Chile) y Peña Nieto (México), dejando en claro el realineamiento de la política exterior argentina, en el ámbito latinoamericano. Además, tal como había promovido en su campaña presidencial, el gobierno avaló la ruptura de la cooperación judicial con Irán determinada por la justicia argentina en diciembre de $2015^{23}$. Sin embargo, no cuestionó la relación con Rusia,

${ }^{22}$ En los primeros días de agosto de 2017, los países de MERCOSUR anunciaron la suspensión de Venezuela como miembro de ese bloque, por tiempo indefinido, y exigieron al presidente Nicolás Maduro restaurar la democracia -desmantelando la Asamblea Constituyente (BBC 2017). Desde su campaña presidencial, Macri fue uno de los promotores de la expulsión de Venezuela del MERCOSUR, debido al incumplimiento de la cláusula democrática por parte de ese país.

23 El 22 de diciembre de 2015, la Cámara Federal de Casación Penal dejó firme el fallo que 
y mucho menos con China -país con el cual continuó la cooperación económica y financiera bilateral. Tampoco se revisaron algunos "acuerdos secretos" que Argentina firmó con China durante la gestión de Cristina F. de Kirchner ${ }^{24}$.

El vuelco en las relaciones de la Argentina con Irán es una de las señales más elocuentes del alineamiento exterior que adoptó Macri. Se encolumnó con Washington. Ya no con el de Obama. Con el de Trump. Una familiaridad que los encargados de su imagen intentan disimular porque exagera ese perfil de empresario antipolítico por el que le hostigan sus opositores. Macri tiene derecho a decir que es ajeno a estos cambios. Que él no controla la justicia. Sólo Netanyahu le jugaría en contra. Cuando le preguntaron [a Netanyahu] si confiaba en la investigación argentina sobre estos temas, no respondió elogiando al Poder Judicial, sino al Poder Ejecutivo: "Macri ha traído un nuevo sentido de dirección e integridad a todo. (Pagni 2017)

La presidencia de Macri también marcó el regreso de la Argentina al Foro Económico Mundial de Davos, en 2016, luego de trece años de ausencia del país en dicho foro. En respuesta al nuevo posicionamiento internacional, la Casa Rosada recibió la visita de numerosos mandatarios extranjeros, en el primer año del gobierno de Cambiemos.

La visita del jefe de la Casa Blanca, Barack Obama, en marzo pasado fue la más destacada de las 15 reuniones bilaterales que se llevaron a cabo en 2016 en la Casa Rosada o en la Quinta de Olivos.

Además del estadounidense, también pasaron por el país el presidente de Francia, François Hollande; los primeros ministros de Japón, Shinzo Abe; de Canadá, Justin Trudeau; y el italiano recientemente renunciado Matteo Renzi [...] Otras reuniones bilaterales que Macri llevó a cabo fueron con los presidentes de Brasil, Michel Temer; de México, Enrique Peña Nieto; de Uruguay, Tabaré Vázquez; de Paraguay, Horacio Cartes; de Bulgaria, Rosen Plevneliev; de Eslovenia, Borut Pahor; el primer ministro de Montenegro, Milo Djukanovic; el emir de Qatar, Sheik Tamin bin Hamad Al Thani; y el gobernador general de Australia, Peter Cosgrove. (Diario Popular 2016)

Otro de los aspectos que la gestión macrista exhibió con orgullo, fue que Argentina fue sede de la reunión (fallida) de la OMC que tuvo lugar en diciembre de 2017 y, sobre todo, el "éxito provisorio" que se logró en la cumbre del G20, desarrollada en diciembre de 2018, en Buenos Aires.

declaraba la inconstitucionalidad del Memorándum firmado entre Argentina e Irán (2013), en el marco de la investigación del atentado a la AMIA (1994), luego de que el gobierno de Macri desistiera de apelar dicho fallo (Télam 2015).

${ }^{24}$ Como es el caso de la misteriosa base que China instaló en la Patagonia argentina (Pérez 2016).
La reformulación de la política exterior argentina en el gobierno de Macri: el difícil equilibrio entre los cambios globales y las crisis domésticas (2015-2018)

Priscila Palacio 


\begin{abstract}
Dossier
América Latina:

continuidad y cambio en el escenario regional
\end{abstract}

Sin embargo, pese a los elogios internacionales que obtuvo la reformulación de la política exterior argentina, la gestión de Macri no consiguió la "lluvia de inversiones" que algunos analistas habían presagiado. Contrariamente, los desequilibrios que venía experimentando la económica argentina desde el año 2012 continuaron durante los primeros años del nuevo gobierno. Incluso, desde el principio, el equipo económico de Macri decidió acudir al financiamiento en el mercado de capitales, incrementando de manera significativa el stock de deuda del país (véase los gráficos a continuación, difundidos por la Secretaría de Finanzas de la República Argentina). Aun así, pese a la creciente emisión de deuda, las políticas domésticas destinadas a "normalizar" (reajustar) la economía, derivaron en una profundización de la estanflación, fundamentalmente, debido al reajuste de las tarifas de servicios públicos. Esas medidas tuvieron fuerte impacto negativo en importantes sectores de la clase media, núcleo principal de apoyo electoral al gobierno Macri.

Gráfico 1.

Deuda Pública Bruta por Moneda en \% del PIB*

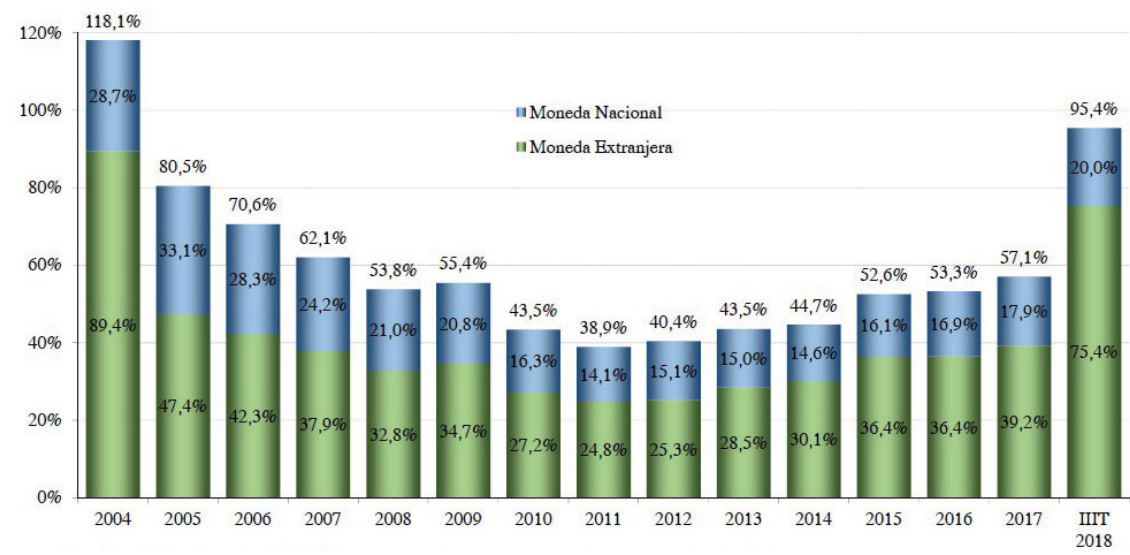

${ }^{*}$ Los datos del PIB publicados por INDEC corresponden a cifras provisorias, provisionales o preliminares. Cifras redondeadas.

Fuente: Secretaría de Finanzas, Ministerio de Hacienda de la República Argentina. Ver en https://www.argentina.gob.ar/hacienda/finanzas/presentaciongraficadeudapublica, fecha de consulta: 04.02.19.

Por otra parte, la emisión de deuda tampoco pudo frenar la especulación cambiaria que proliferó en un mercado de capitales sin controles estatales. Desde mediados de 2017 y durante el 2018, se produjo una estrepitosa depreciación de la moneda nacional (peso), profundizando aún más la crisis económica. El 9.12.12 (día anterior a la asunción de Macri), el tipo de cambio oficial respecto al dólar era 9,736; el 17.12.2015 (días después de la asunción de Macri) se convalidó la depreciación que, de hecho, existía en el mercado 


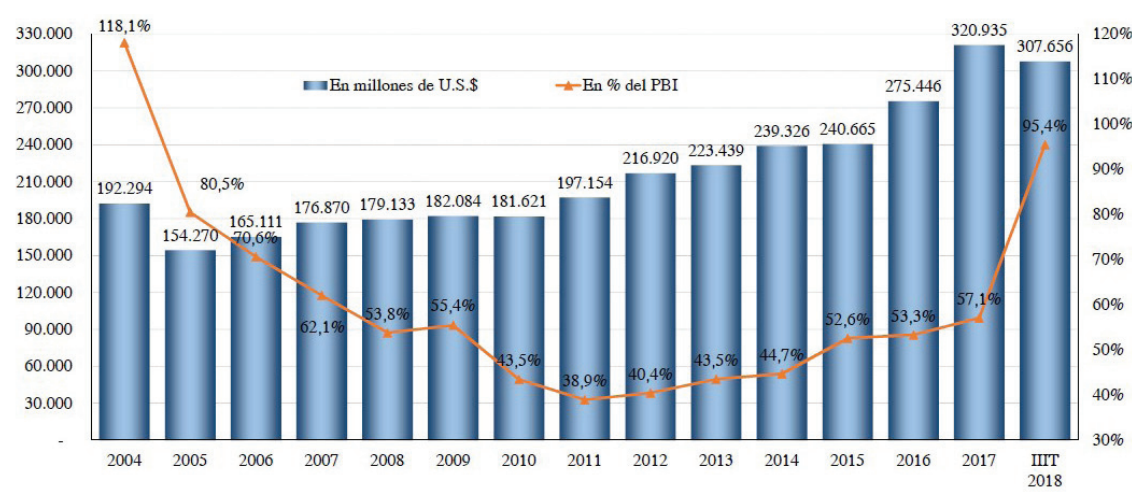

* En la presentación se define a la Deuda Pública Bruta como la deuda performing más la deuda elegible pendiente de reestructuración. Esta última incluye capital, mora de intereses e intereses compensatorios estimados, devengados e impados con posterioridad a la fecha de vencimiento de cada título. Deuda Pública Performing se refiere aquella que se encuentra situación de pago normal.

Fuente: Secretaría de Finanzas, Ministerio de Hacienda de la República Argentina. Ver en https://www.argentina.gob.ar/hacienda/finanzas/presentaciongraficadeudapublica, fecha de consulta: 04.02.19.

de cambios (informal ${ }^{25}$ ), pasando el tipo de cambio a 13,4 pesos por dólar. En mayo de 2018, cuando el gobierno decidió acudir al préstamo de emergencia del FMI, marcó el retorno de la Argentina a dicho organismo, siendo duramente criticado por sectores de la derecha y la izquierda política (Clarín 2018). No obstante, pese a la importante ayuda económica que recibió Argentina del FMI, la especulación prosiguió y el 28.9.2018 el tipo de cambio llegó a 41,25 pesos por dólar (véase gráfico 3 ).

La política económica de la gestión de Macri fue cayendo en un ciclo pernicioso de emisión de deuda, elevadas tasas de interés en el ámbito doméstico, profundización del déficit fiscal y mayor depreciación de la moneda nacional -programa que, en menos de tres años de gobierno, arrasó con un ministro de economía y dos presidentes del Banco Central.

Claramente, la política económica ha sido una de las grandes falencias del gobierno macrista y, en cierto modo, Cambiemos lo atribuyó a la "pesada herencia que dejó el kirchnerismo" y, a los cambios en el contexto internacional que no fueron favorables para los países emergentes. En la segunda mitad de 2018, con la profundización de la crisis económica y las restricciones que impuso el acuerdo del FMI, el gobierno se vio obligado a revisar algunas de sus primeras medidas en política exterior, volviendo a instaurar aranceles a las exportaciones e incluyendo -como novedad- el gravamen
La reformulación de la política exterior argentina en el gobierno de Macri: el difícil equilibrio entre los cambios globales y las crisis domésticas (2015-2018)

Priscila Palacio

\section{Gráfico 2.}

Evolución de la Deuda Pública Bruta* $^{*}$

${ }^{25}$ Denominado mercado "negro o blue", ya que no estaba autorizado su funcionamiento durante el mandato de Cristina F. de Kirchner. 


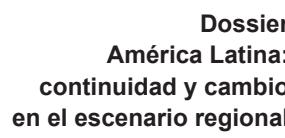

Gráfico 3.

Evolución de la cotización del dólar estadounidense, en pesos

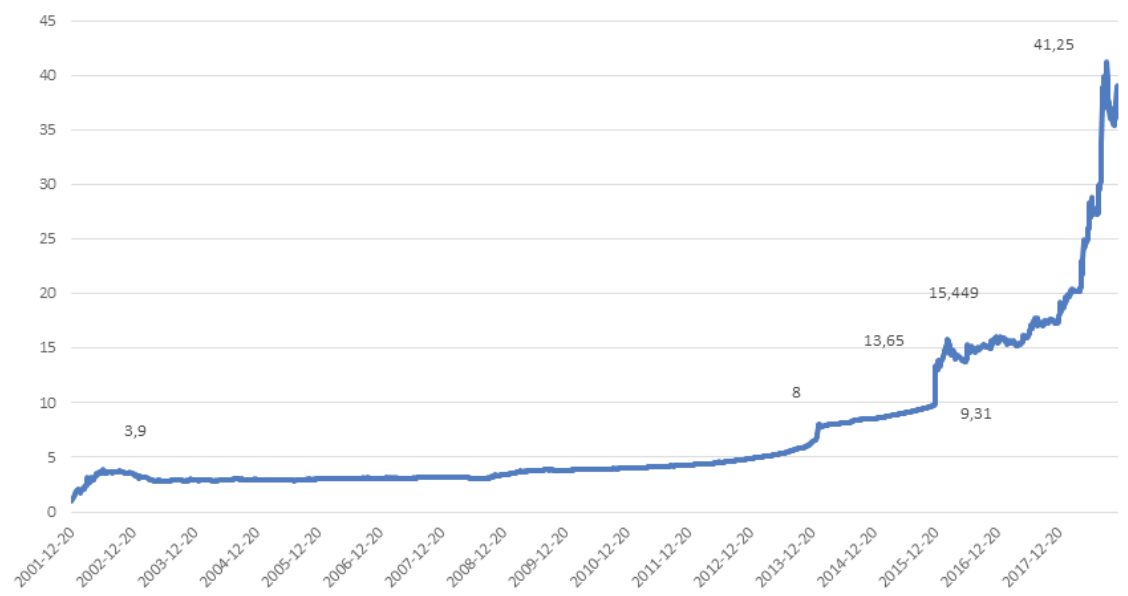

Fuente: elaboración propia sobre la base de datos del Banco Central de la República Argentina. Etiquetas de datos: 3,9 (25/6/02); 8 (9/4/14); 9,31(2/9/15); 13,65 (5/1/16); 15,449 (8/3/16); 41,25 (28/9/18).

a la exportación de servicios (con vigencia a partir de 2019), a fin de equilibrar la Balanza Comercial (BC). Además, aunque el gobierno ha participado en diversas negociaciones para avanzar en la firma de acuerdos de libre comercio, hacia finales del 2018 la liberalización de la economía argentina lejos está de haberse plasmado.

Finalmente, respecto a los sectores sociales más vulnerables, la gestión de Macri revisó y mejoró algunos mecanismos de distribución de recursos, pero continuó con la política de transferencias monetarias no contributivas (planes sociales) que heredó del período de Cristina F. de Kirchner. Incluso, algunos beneficios sociales fueron ampliados para incluir a sectores antes excluidos de esas transferencias monetarias estatales (como la Asignación Universal por Hijo para "monotributistas"). Ello conllevó a que algunos críticos del gobierno tildaran a su gestión de neopopulista. Como señala Alan Greenspan:

El populismo económico busca la reforma, no la revolución. Sus practicantes dejan claro los agravios concretos que hay que corregir, pero sus prescripciones son vagas. A diferencia del capitalismo o el socialismo, el populismo económico no trae consigo un análisis formalizado de las condiciones necesarias para la creación de riqueza y el aumento del nivel de vida. [...] Los líderes populistas ofrecen promesas inequívocas de remediar las injusticias percibidas. [...] En todas sus diversas variedades, por supuesto, el populismo económico lleva la contra al capitalismo de libre mercado. (Greenspan 2008: 378) 
Evidentemente, algunas reformas económicas y de posicionamiento político internacional de Argentina se han ejecutado bajo el gobierno de Macri. Pero la verdadera transformación de la economía argentina, con la erradicación de las restricciones estructurales que tiene la misma, para lograr un crecimiento sustentable e inclusivo en el largo plazo, lejos está de avizorarse.
La reformulación de la política exterior argentina en el gobierno de Macri: el difícil equilibrio entre los cambios globales y las crisis domésticas (2015-2018)

Priscila Palacio

\section{A modo de conclusión}

La política económica exterior que pretendió desarrollar Macri durante su gobierno pareció ir a contramano de las tendencias que prevalecieron en el ámbito global en la segunda década del siglo en curso. Su política defendió la retórica liberal en una coyuntura en la que una parte importante del mundo empezó a cuestionar el neoliberalismo institucional y la globalización. Ello se explica porque la definición de la política exterior argentina, en gran medida, responde a factores domésticos como la ideología de las élites que ejercen poder de lobby en el actual gobierno (las que posiblemente se beneficiarían con la liberalización de la economía nacional).

Sin embargo, pese al importante apoyo internacional que recibió Macri al asumir su gestión, el equilibrio interno y externo que pretendió instaurar su gobierno ha sido difícil de lograr. Si bien se liberalizó el mercado de capitales y se alejó al país de los regímenes tildados de populistas en la región y de otros cuestionados desde el ámbito occidental, el nuevo alineamiento con los Estados Unidos no produjo la "lluvia de inversiones" que el gobierno pretendía obtener. Además de factores externos, sopesó en la decisión de los inversionistas la incertidumbre y crisis de la economía doméstica que ha evidenciado situaciones críticas por el impacto social de las políticas del equipo económico. Incluso, la estanflación que arrastra la economía argentina determinó a que el gobierno debiera mantener importantes egresos por gastos sociales a costa de aumentar la presión fiscal sobre sectores medios de la sociedad (parte de los cuales constituyeron su base de apoyo electoral y se han mostrado desilusionados con la gestión macrista). Tales circunstancias también fueron en desmedro del equilibrio de las cuentas fiscales, reduciendo la capacidad de maniobra del gobierno para enfrentar especulaciones cambiarias en un mercado de cambios liberalizado.

Por otra parte, la contracara del creciente déficit fiscal ha sido el significativo incremento de la deuda externa argentina, así como la incesante problemática de la inflación que ha provocado innumerables conflictos sociales y laborales. Posiblemente, el sector financiero y algunos grupos exportadores estén entre los más beneficiados de la política económica de Macri; no asílos pequeños y medianos empresarios locales (considerando las elevadas tasas de interés que mantiene el BCRA para combatir la inflación). De ese modo, se da la paradoja de que un gobierno tildado de "liberal", termina siendo criticado por liberales acérrimos, debido a la crisis macroeconómica y microeconómica que golpea a vastos sectores de la sociedad argentina. 
Dossier América Latina: continuidad y cambio en el escenario regional
Por lo tanto, es evidente que se han producido varias tensiones en la gestión del gobierno macrista entre 2015 y 2018. Aunque, como dice Gilpin (1981), es difícil dividir el análisis doméstico del internacional, desde el punto de vista analítico puede argumentarse que el gobierno enfrentó en el ámbito doméstico un dilema, entre su aspiración de sostener un idealismo retórico frente a la necesidad de mantener un Estado intervencionista y, en cierto punto, benefactor (con importantes transferencias monetarias no contributivas) que dejó como legado la gestión de Cristina F. de Kirchner. En el ámbito externo, los cambios globales imprimen un pragmatismo que va a contramano de ese idealismo que pretendió instaurar la gestión macrista y que no pudo materializar plenamente en políticas, por el magro desempeño de la economía doméstica (que le obligó a revisar varias de sus primeras medidas de liberalización económica).

Finalmente, el gobierno de Macri se halló ante una encrucijada que le obligó a revisar en la práctica su retórica idealista; una coyuntura global, en la que el liberalismo parece anacrónico, y la necesidad doméstica de mantener un modelo económico (por algunos tildado de) neopopulista, que le ha generado opositores en su propia base electoral.

\section{Referencias bibliográficas}

Arceo E. (2016), La política exterior de Macri: la reprimarización de la economía argentina como objetivo, "Realidad Económica", no 300, 16 de mayo al 30 de junio, Instituto Argentino para el Desarrollo Económico (IADE).

BBC (2017), Mercosur suspende a Venezuela por tiempo indefinido y exige a Maduro que desmantele la Asamblea Constituyente, 05.08.2017, disponible en: https://www.bbc.com/ mundo/noticias-america-latina-40839629, fecha de consulta: 05.05.2018.

Bhagwati J. (2004), In Defense of Globalization, Oxford University Press, New York.

Clarín (2018), Fuertes críticas. La Argentina vuelve al FMI: reacciones tras el anuncio de Mauricio Macri, 08.05.2018, disponible en: https://www.clarin.com/politica/negociacionargentina-fmi-reacciones-despues-anuncio-mauricio-macri_0_Sy2tfI1RM.html, fecha de consulta: 06.08.2018.

Cohen B. J. (2008), International Political Economy. An Intellectual History, Princeton University.

Corradini L. (2011), Los “indignados" se propagan en Europa, "La Nación”, 30.05.2011, disponible en: https://www.lanacion.com.ar/el-mundo/los-indignados-se-propagan-eneuropa-nid1377316, fecha de consulta: 02.05.2018.

Diario Popular (2016), Récord de visitas internacionales en el primer año de Macri, 10.12.2016, disponible en: https://www.diariopopular.com.ar/politica/record-visitasinternacionales-el-primer-ano-macri-n274688, fecha de consulta: 05.06.2018.

Fernández Pardo C. (2015), Zorros y leones: La sociología política de Vilfredo Pareto, Ed. Almaluz, Argentina. 
FMI (2000), La Globalización: ¿Amenaza u Oportunidad?, Fondo Monetario Internacional, https://www.imf.org/external/np/exr/ib/2000/esl/041200s.htm, fecha de consulta: 03.07.2014.

Frieden J. A. (2006), Global Capitalism. Its Fall and Rise in the Twentieth Century, Norton and Company, New York.

Gilpin R. (1981), War and Change in World Politics, Cambridge University Press, [Reimpresión 1999].

Gilpin R. (1990), La Economía política de las Relaciones Internacionales, trad. por Cristina Piña, Grupo Editor Latinoamericano, Buenos Aires. Título original en inglés The Political Economy of International Relations, Princeton University Press [1987].

Greenspan A. (2008), La era de las turbulencias. Aventuras en un nuevo mundo, Ediciones B.S.A., Barcelona.

iProfesional (2016), Lo esperamos como primer caballero en el G20 en Buenos Aires de 2018, le dijo el Presidente al ex mandatario norteamericano, 26.09.2016, disponible en: https:// www.iprofesional.com/notas/239200-mauricio-macri-hillary-clinton-bill-clinton-Macriapoyo-la-candidatura-de-Hillary-Clinton-con-un-fuerte-mensaje-a-su-esposo, fecha de consulta: 10.05.2018.

Kirshner J. (2010), Realist Political Economy: Traditional Themes and Contemporary Challenges, en: Routledge Handbook of International Political Economy (IPE). IPE as a global conversation, M. Blyth (ed.), Routledge.

La Nación (2015), Confirmado: Macri anunció retenciones cero, salvo para la soja, 14.12.2015, disponible en: https://www.lanacion.com.ar/economia/mauricio-macri-presidente-anunciarecortes-retenciones-al-campo-nid1854066, fecha de consulta: 05.07.2018.

Mastanduno M. (2012), The Richness of the Contributions of Robert G. Gilpin, en: Robert Gilpin \& International Relations. Reflections, W. Danspeckgruber (comp.), Liechtenstein Colloquium Report, vol. 2, USA.

Mearsheimer J. J. (2006), Structural Realism, en: International Relations Theories: Discipline and Diversity, T. Dunne, M. Kurki, S. Smith (eds.), Oxford University Press.

Ocampo J. A. (2009), Impactos de la crisis financiera mundial sobre América Latina, "Revista CEPAL", no 97, abril.

Olesen T. (2004), The Transnational Zapatista Solidarity Network: An Infrastructure Analysis, "Global Networks", no 4(1), Blackwell Publishing Ltd \& Global Networks Partnership, pp. 89-107.

O'Neill J. (2001), Building Better Global Economic BRICs, Global Economics, Paper no 66, 30th November 2001, GS Global Economics Website, Goldman Sachs, disponible en: https://www.gs.com, fecha de consulta: 27.05.2014.

Pagni C. (2017), Irán: Macri gira igual que Trump, "El País", 16.10.2017, disponible en: https://elpais.com/internacional/2017/10/16/america/1508184058_834958.html, fecha de consulta: 10.07.2018.

Palacio P. (2008), La globalización financiera y el nuevo orden mundial, en: Historia Económica y Social del Mundo Contemporáneo. Ejes para su comprensión, M. de M. Llairó, M. Díaz (coords.), Ed. Errepar, Argentina.

Palacio P. (2012), Crisis financiera y hegemónica internacional. Sus consecuencias para los países emergentes y menos desarrollados, en: Los dilemas de América Latina ante las crisis. Conflictos $y$ alternativas de desarrollo, M. de M. Llairó, P. Palacio (comps.), Ed. Eudeba, Argentina.
La reformulación de la política exterior argentina en el gobierno de Macri: el difícil equilibrio entre los cambios globales y las crisis domésticas (2015-2018)

Priscila Palacio 
Dossier América Latina: continuidad y cambio en el escenario regional
Palacio P. (2019), Realismo y cambio internacional: estudio sobre la obra de Robert Gilpin War and Change y su contemporaneidad en el siglo XXI: una mirada a Estados Unidos, China y Rusia, Editorial Almaluz, Argentina.

Pérez C. (2016), Lo que se sabe de la misteriosa base que China está construyendo en la Patagonia argentina, BBC, 17.03.2016, disponible en: https://www.bbc.com/mundo/ noticias/2016/03/160317_misteriosa_base_china_patagonia_argentina_lb, fecha de consulta: 06.08.2018.

Rourke J. T. (2007), International Politics on the World Stage, Twelfth Edition, University of Connecticut, Mc Graw Hill, Boston.

Snyder J. (1991), Myths of Empire. Domestic Politics and International Ambition, Cornell University, Ithaca and London.

Télam (2015), Quedó firme la inconstitucionalidad del memorándum de entendimiento con Irán, 22.12.2015, disponible en: http://www.telam.com.ar/notas/201512/130942-quedofirme-la-inconstitucionalidad-del-memorandum-de-entendimiento-con-iran.html, fecha de consulta: 05/05/2018.

Tokatlian J. G., Russell R. (2017), Macri: en busca de una nueva inserción internacional, “Anuario Internacional CIDOB 2016-17", disponible en: https://www.raco.cat/index.php/ AnuarioCIDOB/article/view/348683/439917, fecha de consulta: 06.10.2018.

Valli P. (2016), Macri les pagó a los buitres y la Argentina salió del default, "Perfil”, 23.04.2016, disponible en: https://www.perfil.com/noticias/economia/macri-les-pago-a-los-buitres-yla-argentina-salio-del-default-0422-0102.phtml, fecha de consulta: 07.04.2018.

\section{Otras fuentes consultadas}

Banco Central de la República Argentina

Secretaría de Finanzas, Ministerio de Hacienda de la República Argentina, disponible en: https://www.argentina.gob.ar/hacienda/finanzas/presentaciongraficadeudapublica, fecha de consulta: 04.02.19. 